\title{
SATISFAÇÃO PROFISSIONAL E CULTURA ORGANIZACIONAL: UM ESTUDO DE CASO EM UMA INSTITUIÇÃO FEDERAL DE ENSINO
}

\author{
E. PEREIRA FILHO ${ }^{1}$, C. C. A. FLORENCIO ${ }^{1}$, N. M. C. P. G. LEONE ${ }^{2}$ \\ ${ }^{1}$ Instituto Federal do Rio Grande do Norte - IFRN \\ ${ }^{2}$ Universidade Potiguar - UnP \\ evadio.filho@ifrn.edu.br
}

Artigo submetido em março/2014 e aceito em agosto/2014

DOI: $10.15628 /$ holos.2014.2073

\section{RESUMO}

O presente artigo tem como objetivo investigar, na perspectiva dos servidores técnico-administrativos do Instituto Federal de Educação, Ciência e Tecnologia do Rio Grande do Norte (IFRN), a relação entre satisfação e cultura organizacional, com base no modelo ASH. Para tanto, foram entrevistados, mediante questionários, 307 sujeitos. Dentre os achados, houve a identificação de quatro dimensões da satisfação profissional, assim como seis fatores que retratam os estilos de cultura. Percebese, contudo, uma conformidade entre as dimensões encontradas neste estudo e aquelas presentes nos escritos de Quijano e Navarro (1999) e Barboza (2008).
Ademais, nota-se, mesmo que de magnitude fraca ou moderada, a existência de correlação significativa entre cultura organizacional e a satisfação. Constatou-se que as quatro dimensões da satisfação estão relacionadas com pelo menos algum estilo de cultura - exceto convencional-tarefa e normativo, os quais esta associação não foi evidenciada. Os resultados permitem concluir que a variável satisfação está associada à cultura organizacional. No entanto, não foi objeto de estudo apontar qual o sentido da correlação, ou seja, qual dentre elas é a antecedente e qual é a consequente

PALAVRAS-CHAVE: Cultura organizacional, satisfação, modelo ASH.

\section{PROFESSIONAL SATISFACTION AND ORGANIZATIONAL CULTURE: A CASE STUDY IN A FEDERAL EDUCATION INSTITUTION}

\begin{abstract}
This paper aims to investigate, from the perspective of technical and administrative staff of the Federal Institute of Education, Science and Technology of Rio Grande do Norte (IFRN), the relationship between satisfaction and organizational culture, based on the ASH model. For both, were interviewed through questionnaires 307 subjects. Among the findings was the identification of four dimensions of job satisfaction, as well as six factors that depict the styles of culture. It is clear, however, a line between the dimensions found in this study and those found in the writings of Quijano and Navarro (1999) and
\end{abstract}

Barbosa (2008). Moreover, there is, even if weak or moderate magnitude, the existence of a significant correlation between organizational culture and satisfaction. It was found that the four dimensions of satisfaction are related to at least some style culture except conventional-task and normative, which this association was not established. The results indicate that the satisfaction variable is associated with organizational culture. However, it was not the object of study point where the direction of this correlation, i.e., which of them is the antecedent and what is the consequent.

KEYWORDS: Organizational culture, satisfaction, ASH model. 


\section{INTRODUÇÃO}

Obter um melhor desempenho organizacional perpassa pela compreensão dos valores, crenças e demais elementos que compõem a cultura organizacional (GIBSON et al., 2006). É necessário existir um alinhamento entre os aspectos macro (organização) e micro (indivíduo), de forma que essa consonância reflita positivamente no desempenho da organização. Para Goffee e Jones (1998), a cultura organizacional é vista como o paradoxo dos negócios, por ser tão criticamente ligada ao sucesso, mas, ao mesmo tempo, tão suave, nebulosa e indefinível. Nota-se que receitas e lucros são perseguidos, a taxa de retenção de clientes é avaliada, a participação no mercado é calculada, no entanto, o impacto da cultura de uma empresa sobre o seu desempenho, somente pode ser inferida. Logo, para aqueles que almejam o sucesso de sua estratégia ou plano de negócio, é interessante que a cultura não seja menosprezada. Pois, desconsiderá-la é deixar ao relento um grupo de valores e normas que norteiam as interações dos membros internos e externos da organização.

A satisfação profissional, por sua vez, leva a um aumento da produtividade da organização (MARTINS; SANTOS, 2006). Funcionários satisfeitos são trabalhadores produtivos; estão mais propensos a falar bem da organização, a colaborar com os demais e a superar as expectativas em relação ao seu trabalho. Ademais, funcionários satisfeitos tornam-se mais dispostos a ir além de suas atribuições regulares, porque querem retribuir suas experiências positivas. Para Quijano e Navarro (1999), os baixos níveis de satisfação conduzem as pessoas a boicotar e a render menos no trabalho. Atualmente, o capital humano configura-se como um elemento chave no êxito dos negócios. Sem uma gestão adequada dos recursos humanos, as empresas terão dificuldades em executar suas metas e objetivos, e, consequentemente, não atingiram os resultados almejados.

Conforme Martins e Santos (2006), conteúdos mentais do indivíduo como crenças, valores, fatores disposicionais, moral e a possibilidade de desenvolvimento no trabalho são preditores da satisfação. Face às reflexões supracitadas, suscita a seguinte questão de pesquisa: como a satisfação profissional relaciona-se com a cultura organizacional de uma Instituição Federal de Ensino?

Portanto, o presente trabalho tem como objetivo investigar, na perspectiva dos servidores técnico-administrativos do Instituto Federal de Educação, Ciência e Tecnologia do Rio Grande do Norte (IFRN), a relação entre satisfação e cultura organizacional, com base no modelo intitulado Auditoria do Sistema Humano (ASH) proposto por Quijano e Navarro (1999). Foram entrevistados, mediante questionários, 307 sujeitos. O cálculo amostral considerou o número de técnicoadministrativos ativos, no momento da realização da pesquisa a qual foi realizada em meados de novembro 2012. Em termos de implicações gerenciais, os resultados podem ser usados para dar suporte às decisões dos gestores de aperfeiçoamento das estratégias de recursos humanos.

\section{FUNDAMENTAÇÃO TEÓRICA}

\subsection{CULTURA ORGANIZACIONAL}

A conceituação de cultura tem suas raízes nas áreas da antropologia e da sociologia, e com o decorrer do tempo alcançou o âmbito das organizações. Foi a partir de então que começou a ser 
utilizada na explicação das diferenças de desempenho, análise da globalização dos mercados e no estudo do comportamento dos membros organizacionais (BERTERO, 1996). Segundo Dornelas et al. (2009), no início da década de 50, Elliot Jaques foi um dos pioneiros no uso do termo cultura inserido no contexto organizacional. Ao conjunto de ideias e ações, mais ou menos compartilhadas, a serem aprendidas e assumidas pelos novos integrantes da empresa para que fossem aceitos; ele denominou "cultura da empresa".

Para Aktouf (1994), a cultura representa um

[...] conjunto complexo e multidimensional de praticamente tudo o que constitui a vida em comum nos grandes grupos sociais. [...] Uma maneira diferente de designar um sistema informal generalizado, irracional e talvez, profundamente subjetivo ou inconsciente. (AKTOUF, 1994, pp. 40-42).

Segundo Schein (1985), consiste em um bojo de suposições que ensina a lidar com problemas externos de adaptação e internos de integração, e pode ser considerado válido a ponto de ser repassado aos novos membros do grupo. Cameron e Quinn (1999), por sua vez, apesar de identificarem na cultura elementos de diferenciação e fragmentação, consideram que a sua força está na habilidade de fazer as pessoas permanecerem unidas, de forma a superar a fragmentação e a ambiguidade, tão peculiares ao ambiente externo, conduzindo a empresa na direção do sucesso. Para esses autores, a cultura é percebida como uma vantagem competitiva das organizações à medida que representa um conjunto de percepções, memórias, valores, atitudes e definições geradas por consenso e, portanto, comuns, além de integradas. Entendem a cultura como sendo algo gerenciável e, portanto, passível de mudança. Robbins (2005) coaduna com este pensamento, ao afirmar que a cultura organizacional refere-se a um sistema de valores, compartilhado pelos membros de um grupo, o qual diferencia uma organização das demais.

Neste estudo, o entendimento de cultura organizacional está albergado no modelo ASH, de autoria de Quijano e Navarro (1999). Nele, enumeram-se cinco dimensões ou estilos culturais. A seguir estão descritos os estilos culturais, juntamente com as respectivas variáveis que os compõem:

- Estilo de Realização de Pessoas: compreendem as culturas em que há o favorecimento às relações interpessoais positivas, a satisfação coletiva, o estímulo ao crescimento pessoal e o alcance de objetivos ambiciosos, porém realistas. Este estilo de cultura apresenta as seguintes subdimensões (subcategorias): autorrealização, apoio a pessoas, necessidade de crescimento (necessidade de realização) e afiliação.

- Estilo de Poder-Evitação: representam as culturas que enaltecem as relações políticas entre seus membros, buscando a obtenção de poder e incentivando a oposição indireta dos membros às coisas que se levam a cabo e ao senso de responsabilidade dos demais. Sendo caracterizada pelas seguintes subdimensões: evitação, oposição e poder.

- Estilo Convencional-Dependente: há o incentivo ao acatamento das ordens sem questionamento algum e são apoiadas as relações com bases no convencionado de acordo com a ordem estabelecida e estimulam as relações em que se busca a 
aprovação dos demais. São divididas através das seguintes subdimensões: convencional-social, dependente e aprovação.

- Estilo Convenção Tarefa-Perfeccionismo: pode ser caracterizado pelo estímulo a fazer as coisas como sempre se tenha feito e a se preocupar em realizar controle sobre os erros, havendo atitude de perfeccionismo em tudo que se faz. Suas subcategorias são: convencional-tarefa e perfeccionista.

- Estilo Competitivo: são culturas que favorecem as relações de competição interpessoal do tipo ganhar-perder e as pessoas são recompensadas por superar os demais.

\subsection{SATISFAÇÃO PROFISSIONAL}

A satisfação no trabalho é reconhecida como um fenômeno complexo e de difícil significado. Isso se deve ao fato de ser considerado um estado subjetivo e pessoal percebido pelo homem em relação ao que ocorre a seu favor. Resulta da avaliação que o indivíduo faz de seu trabalho, bem como da percepção como se satisfaz ou permite satisfazer-se pelos valores agregados ao trabalho (MARTINEZ, 2002).

Muitos fatores explicam por que os profissionais permanecem ou não numa organização. A satisfação no trabalho é um dos fatores mais pesquisados na tentativa de explicar tal fenômeno, principalmente o que diz respeito a sua correlação com a rotatividade. A literatura aponta o nível de insatisfação do funcionário com relação à função que desempenha como um fator determinante que o leva a desvincular-se de uma organização. Descontentamento, esse, que pode ser originado por qualquer um dos inúmeros aspectos que compõem o trabalho. A existência da relação negativa entre satisfação no trabalho e rotatividade foi largamente comprovada em estudos empíricos (HERZBERG, 1964; VROOM, 1964; LÉVI-LEBOYER, 1994; ROBBINS, 2005).

Conforme Robbins (2005) o termo satisfação com o trabalho é definido como uma atitude geral do indivíduo em relação ao trabalho que realiza. Deste modo, uma pessoa com alto nível de satisfação apresenta atitudes positivas em relação ao trabalho, ao passo que, aquela insatisfeita apresenta atitudes negativas. E na arte de mensuração este conceito, não se deve levar em consideração apenas as atividades corriqueiras, mas também a convivência com colegas e superiores, a obediência às regras e políticas organizacionais, o alcance de padrões de desempenho, a aceitação de condições de trabalho e outras coisas do gênero. A avaliação que um funcionário faz de sua (in)satisfação com o trabalho é resultante de um complexo somatório de diferentes elementos. $O$ autor ressalta, ainda, que a cultura organizacional afeta o desempenho e a satisfação dos funcionários, sendo maior impacto quanto mais forte é a cultura. Para ele, pessoas cujos valores não se ajustam aos da empresa mostram-se pouco motivados e com baixo comprometimento, sentindo-se insatisfeitos com seu trabalho e com a organização (ROBBINS, 2005).

Para este estudo, a satisfação profissional baseia-se no modelo ASH, proposto por Quijano e Navarro (1999), o qual compreende as variáveis: retribuição (salário e forma de pagamento); condições físicas ao redor do trabalho; seguridade e estabilidade do emprego; relação com os companheiros; relação com os superiores; reconhecimento pelo trabalho bem feito; autorrealização (satisfação pessoal do funcionário por se desenvolver no trabalho, pela sua 
aprendizagem e adequação de seu trabalho a sua formação e interesses profissionais); benefícios sociais e nível de satisfação geral.

\subsection{AUDITORIA DO SISTEMA HUMANO - MODELO ASH}

O Comportamento Organizacional é visto como um dos campos de estudo de expressiva importância dentro das ciências sociais, com vista ao bom desenvolvimento das empresas. Investiga as consequências que indivíduos, grupos e a estrutura podem trazer ao desempenho das organizações, utilizando esse conhecimento para a melhoria da eficácia organizacional (ROBBINS, 2005).

$\mathrm{Na}$ busca por essa excelência na prestação de seus serviços, as organizações vêm desenvolvendo métodos capazes de avaliar e observar de que forma as pessoas têm contribuído para o alcance da efetividade organizacional. Um desses métodos, elaborado por Quijano e Navarro, em 1999, na Universidade de Barcelona, é chamado de Auditoria do Sistema Humano (ASH). O objetivo é o de diagnosticar e avaliar a Gestão Estratégica de Recursos Humanos nas empresas, tendo, desde o início, uma clara intenção de avaliação para a intervenção orientada à efetividade organizacional (QUIJANO; NAVARRO, 1999).

De acordo com Oliveira (2004), o referido modelo avalia a organização, a partir dos aspectos do ambiente onde está inserida, bem como dos seus aspectos estratégicos, do desenho organizacional (tecnologia, estrutura e modelo de gestão de pessoas) e de seus processos psicológicos e psicossociais dos indivíduos envolvidos na organização. Procura compreender como administrar pessoas e com pessoas. Em síntese, o autor afirma ser necessário avaliar como a qualidade dos recursos humanos contribui para o alcance da efetividade organizacional. A Figura 1 auxilia na compreensão do modelo ASH.

\begin{tabular}{|c|c|c|c|c|c|c|}
\hline \multirow{3}{*}{ AMBIENTE } & \multicolumn{9}{|c|}{ A ORGANIZAÇÃO } \\
\cline { 3 - 4 } & \multirow{3}{*}{ ESTRATÉGIA } & \multicolumn{3}{|c|}{ DESENHO } & \multicolumn{2}{|c|}{$\begin{array}{c}\text { EFETIVIDADE } \\
\text { ORGANIZACIONAL }\end{array}$} \\
\cline { 3 - 5 } \cline { 5 - 7 } & Tecnologias & Estrutura & $\begin{array}{c}\text { Sistema } \\
\text { de } \\
\text { Gestão }\end{array}$ & PROCESSOS & $\begin{array}{c}\text { Sobre as } \\
\text { pessoas }\end{array}$ & $\begin{array}{c}\text { Sobre as } \\
\text { organizações }\end{array}$ \\
\hline
\end{tabular}

\begin{tabular}{|c|c|c|c|c|c|c|c|}
\hline 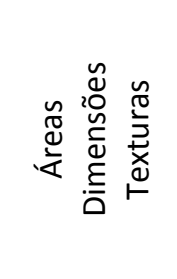 & 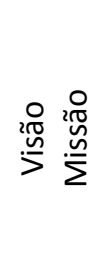 & 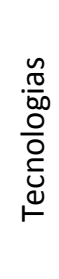 & 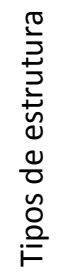 & 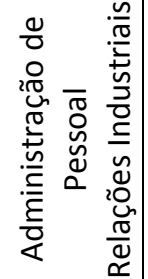 & 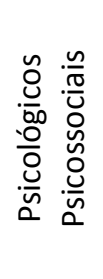 & $\begin{array}{l}\frac{1}{0} \\
\frac{1}{n} \\
\frac{0}{0} \\
0 \\
\frac{0}{0} \\
\frac{\pi}{0} \\
\frac{0}{0} \\
\frac{0}{2}\end{array}$ & 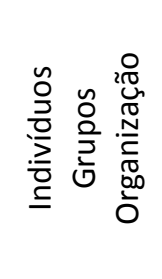 \\
\hline
\end{tabular}

Figura 1 - Modelo de Auditoria do Sistema Humano. Fonte: OLIVEIRA (2004). 


\section{PROCEDIMENTOS METODOLÓGICOS}

O trabalho de pesquisa foi balizado pela literatura que versa sobre satisfação e cultura organizacional. O esquema metodológico adotado no desenvolvimento da pesquisa é apresentado na Figura 2.

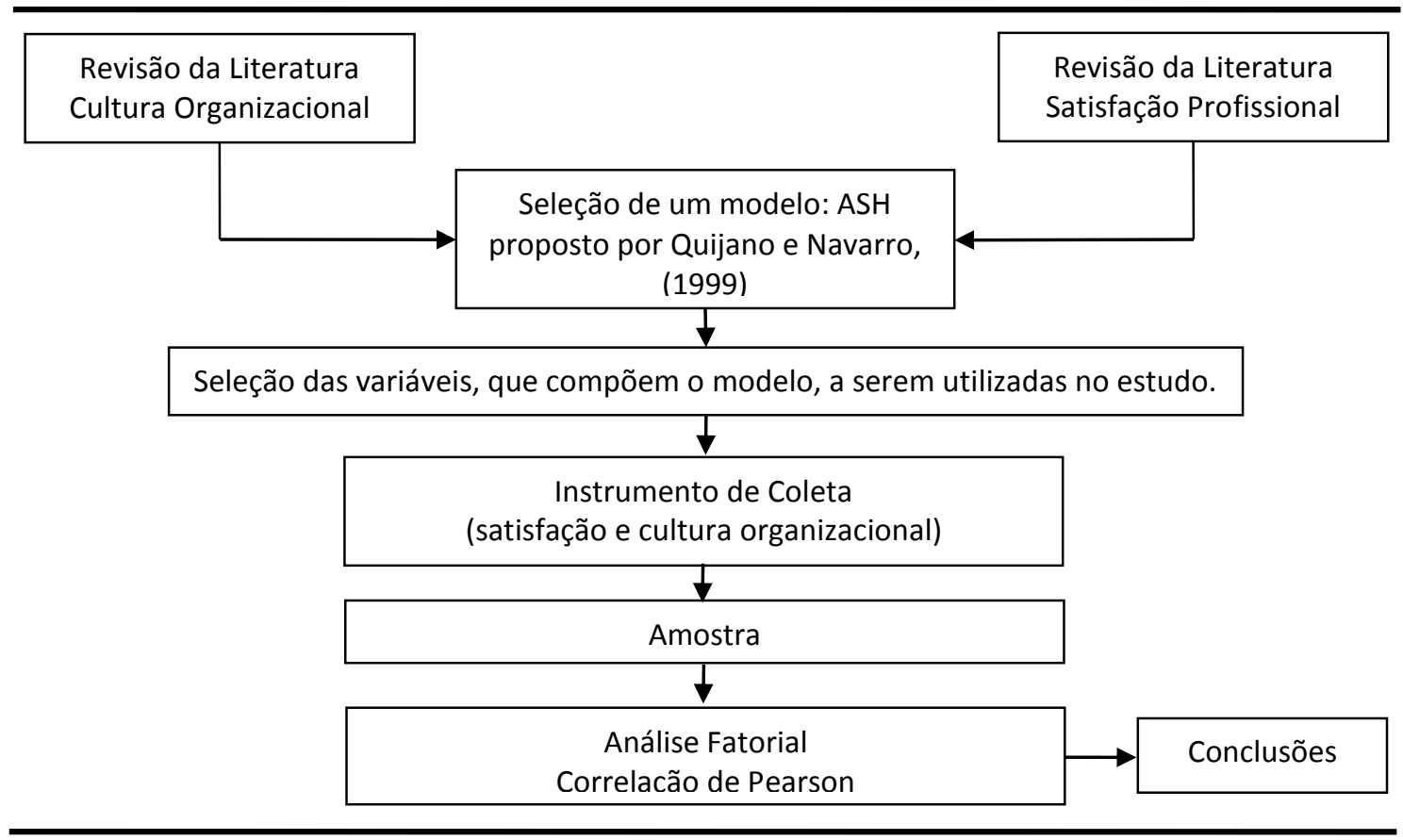

Figura 2 - Esquema metodológico.

Fonte: Elaborado pelos Autores

\subsection{TIPO DE PESQUISA}

A presente investigação caracteriza-se como descritiva, com abordagem quantitativa, além de uma pesquisa de campo com estudo de caso. $O$ traço descritivo está fundamentado no ato do pesquisador em analisar e correlacionar fatos sem manipulá-los, com intuito de conhecer as situações e relações aos aspectos do comportamento humano. Ademais, o cunho quantitativo do trabalho fundamenta-se no ato de capturar o grau de satisfação dos funcionários e a percepção dos servidores em relação ao nível de importância que a empresa confere a elementos do modelo ASH. Em termos de pesquisa de campo com estudo de caso, o motivo que conduz a esta classificação alicerça-se na capacidade de analisar ampla e detalhadamente a questão de pesquisa no âmbito de uma realidade específica: o Instituto Federal de Educação, Ciência e Tecnologia do Rio Grande do Norte (OLIVEIRA, 2002; VERGARA, 2006; GIL, 2010).

\subsection{POPULAÇÃO E AMOSTRA}

A população foi constituída pelos 788 técnicos-administrativos pertencentes ao quadro ativo do Instituto Federal de Educação, Ciência e Tecnologia do Rio Grande do Norte - IFRN, distribuídos em seus 15 campus e Reitoria. 
A amostra apresentou um caráter não probabilístico, sendo os respondentes selecionados por critérios de conveniência e acessibilidade. Atingiu a grandeza de 297 servidores, admitindo-se um erro de 4,5\% e um nível de confiança de 95\%, conforme detalhado na Tabela 1.

Tabela 1 - População e amostra.

\begin{tabular}{cccc}
\hline Organização & População & Amostra* & Quantidade planejada \\
\hline IFRN & 788 & 297 & 312 \\
\hline
\end{tabular}

Fonte: Pesquisa (2012). (*) 0 tamanho da amostra foi calculado admitindo-se um erro de 4,5\% e um nível de confiança de $95 \%$.

Os pesquisadores optaram por exceder, na grandeza de 15 unidades, o quantitativo de questionários a ser aplicado, estimando, assim, 312 respondentes. Todavia, 307 questionários foram válidos por apresentarem todas as questões assinaladas, com indicação de uma única resposta.

\subsection{VARIÁVEIS ANALÍTICAS UTILIZADAS}

O conjunto de variáveis analíticas utilizado no estudo foi retirado do modelo ASH, proposto por Quijano e Navarro (1999), dentre os 581 itens que o integram. Estas foram selecionadas em razão dos pesquisadores: julgarem apropriadas e aplicáveis ao recorte espacial analisado; pertinentes às dimensões estudadas: satisfação e cultura organizacional; e por estarem consolidadas no estudo de Barboza (2008). A Figura 3 mostra as variáveis relacionadas à dimensão satisfação profissional e em qual subdimensão "necessidades" cada uma está enquadrada.

\begin{tabular}{|c|c|c|}
\hline SG. & Variáveis & Necessidades \\
\hline S01. & O respeito que lhe oferecem os companheiros com os quais trabalha & Relação com os companheiros \\
\hline S02. & As relações que mantém com seus companheiros de trabalho & Relação com os companheiros \\
\hline S03. & A possibilidade de fazer amigos no trabalho & Relação com os companheiros \\
\hline S04. & O apoio que recebe por parte de seus superiores imediatos & Relação com os superiores \\
\hline S05. & O respeito que lhe oferecem seus superiores & Relação com os superiores \\
\hline S06. & O reconhecimento que recebe, por parte de seus superiores, quando merece & $\begin{array}{c}\text { Reconhecimento pelo trabalho } \\
\text { bem feito }\end{array}$ \\
\hline S07. & Os elogios que recebe, quando obtém bons resultados & $\begin{array}{c}\text { Reconhecimento pelo trabalho } \\
\text { bem feito }\end{array}$ \\
\hline S08. & As possibilidades de aprender e desenvolver-se profissionalmente & Autorrealização \\
\hline so9. & $\begin{array}{l}\text { O nível de conhecimentos e habilidades que pode pôr em jogo no posto de } \\
\text { trabalho }\end{array}$ & Autorrealização \\
\hline S10. & A adequação de seu trabalho à sua formação e interesses profissionais & Autorrealização \\
\hline S11. & O salário que recebe & Retribuição \\
\hline S12. & A forma como lhe pagam & Retribuição \\
\hline S13. & A segurança no emprego, frente ao futuro & $\begin{array}{l}\text { Segurança e estabilidade no } \\
\text { emprego }\end{array}$ \\
\hline S14. & O nível de segurança e estabilidade que lhe oferece a empresa & $\begin{array}{c}\text { Segurança e estabilidade no } \\
\text { emprego }\end{array}$ \\
\hline S15. & As condições físicas no seu ambiente de trabalho & $\begin{array}{l}\text { Condições físicas do ambiente } \\
\text { do trabalho }\end{array}$ \\
\hline S16. & O ambiente físico de seu posto de trabalho & $\begin{array}{c}\text { Condições físicas do ambiente } \\
\text { do trabalho }\end{array}$ \\
\hline
\end{tabular}




\begin{tabular}{|c|c|c|}
\hline S17. & $\begin{array}{c}\text { Os benefícios sociais que a empresa lhe oferece (tíquetes de estaurante, } \\
\text { plano de saúde...). }\end{array}$ & Benefícios sociais \\
\hline S18. & $\begin{array}{c}\text { Em geral, considerando todos os aspectos anteriores, com este emprego } \\
\text { você está... }\end{array}$ & Satisfação em geral \\
\hline
\end{tabular}

Figura 3 - Variáveis relacionadas à dimensão satisfação profissional Fonte: Adapatado de Quijano e Navarro (1999). Legenda: SG - sigla correspondente a variável.

A Figura 4 retrata as variáveis relativas à dimensão cultura organizacional e em qual estilo cada uma está inserida.

\begin{tabular}{|c|c|c|}
\hline SG. & VARIÁVEIS & Estilos da Cultura Organizacional \\
\hline Q1 & Estejam atentos aos erros dos demais & Poder Evitação (Oposição) \\
\hline Q2 & Ajudem aos demais a crescerem e desenvolverem-se & Realização de Pessoas (Apoio a pessoa) \\
\hline Q3 & Se comportem como "bons moços" & Convencional-Dependente (Aprovação) \\
\hline Q4 & Tendam a competir mais que a colaborar & Competitivo \\
\hline Q5 & Assumam tarefas que envolvam desafios & $\begin{array}{l}\text { Realização de Pessoas (Necessidade de } \\
\text { Realização) }\end{array}$ \\
\hline Q6 & Aceitem os objetivos sem questioná-los & Convencional-Dependente (Dependente) \\
\hline Q7 & Tratem os demais de modo agradável e amistoso & Realização de Pessoas (Afiliação) \\
\hline Q8 & Se preocupem em obter poder & Poder Evitação (Poder) \\
\hline Q9 & Nunca cometam erros & $\begin{array}{c}\text { Convencional Tarefa-Perfeccionismo } \\
\text { (Perfeccionismo) }\end{array}$ \\
\hline Q10 & Façam as coisas como sempre fizeram & Convencional-Tarefa (Tarefa-Convencional) \\
\hline Q11 & Adaptem suas opiniões para evitar conflitos & $\begin{array}{c}\text { Convencional-Dependente (Convencional- } \\
\text { Social) }\end{array}$ \\
\hline Q12 & Esperem que os demais ajam primeiro & Poder-Evitação (Evitação) \\
\hline Q13 & Gostem de seu trabalho & Realização de Pessoas (Autorrealização) \\
\hline Q14 & Se oponham indiretamente às coisas & Poder-Evitação (Oposição) \\
\hline Q15 & Dediquem tempo aos outros & Realização de Pessoas (Apoio a pessoa) \\
\hline Q16 & Sejam conscientes da realidade da empresa & Convencional-Dependente (Aprovação) \\
\hline Q17 & Sejam "vencedores" & Competitivo \\
\hline Q18 & Prevejam e planejem & $\begin{array}{c}\text { Realização de pessoas (Necessidade de } \\
\text { Realização) }\end{array}$ \\
\hline Q19 & Cumpram as ordens, mesmo que sejam equivocadas. & Convencional-Dependente (Dependente) \\
\hline Q20 & Pensem em termos da satisfação do grupo & Satisfação Pessoal (Afiliação) \\
\hline Q21 & $\begin{array}{l}\text { Se movam "politicamente" para ganhar influência entre } \\
\text { os funcionários }\end{array}$ & Poder Evitação (Poder) \\
\hline Q22 & Sejam precisos, mesmo quando não seja necessário & $\begin{array}{l}\text { Convencional Tarefa-Perfeccionismo } \\
\text { (Perfeccionismo) }\end{array}$ \\
\hline Q23 & Não mudem o caminho habitual de proceder & $\begin{array}{c}\text { Convencional Tarefa-Perfeccionismo } \\
\text { (Convencional- Tarefa) }\end{array}$ \\
\hline Q24 & Aceitem a ordem estabelecida & $\begin{array}{l}\text { Convencional-Dependente (Convencional- } \\
\text { Social) }\end{array}$ \\
\hline Q25 & Adiem as coisas & Poder-Evitação (Evitação) \\
\hline Q26 & Divulguem suas ideias & Realização de Pessoas (Autorrealização) \\
\hline Q27 & $\begin{array}{l}\text { Questionem habitualmente as decisões tomadas por } \\
\text { outros }\end{array}$ & Poder-Evitação (Oposição) \\
\hline Q28 & Ajudem os demais a pensar por si mesmos & Realização de Pessoas (Apoio a pessoa) \\
\hline Q29 & Tenham fácil contato com todos & Realização de Pessoas (Afiliação) \\
\hline Q30 & Mantenham uma imagem de superioridade & Poder Evitação (Poder) \\
\hline Q31 & Assumam riscos altos, embora alcançáveis & $\begin{array}{l}\text { Realização de Pessoas (Necessidade de } \\
\text { Realização) }\end{array}$ \\
\hline Q32 & Obedeçam às ordens de boa vontade & Convencional-Dependente (Dependente) \\
\hline Q33 & Sejam abertos e calorosos & Realização de Pessoas (Afiliação) \\
\hline
\end{tabular}




\begin{tabular}{|c|c|c|}
\hline Q34 & Preservem a autoridade sem questioná-la & Convencional-Dependente (Dependente) \\
\hline Q35 & Atuem buscando a perfeição & $\begin{array}{l}\text { Convencional Tarefa-Perfeccionismo } \\
\text { (Perfeccionismo) }\end{array}$ \\
\hline Q36 & $\begin{array}{l}\text { Se restrinjam sempre às regras e procedimentos } \\
\text { estabelecidos }\end{array}$ & $\begin{array}{l}\text { Convencional Tarefa-Perfeccionismo } \\
\text { (Convencional- Tarefa) }\end{array}$ \\
\hline Q37 & Se adaptem ao "modelo esperado" & Convencional-Dependente (Aprovação) \\
\hline Q38 & Não gostem das decisões que saem de baixo para cima & Poder-Evitação (Evitação) \\
\hline Q39 & Pensem de maneira original e independente & Realização de Pessoas (Autorealização) \\
\hline
\end{tabular}

Figura 4 - Variáveis relacionadas à dimensão cultura organizacional

Fonte: Adapatado de Quijano e Navarro (1999). Legenda: SG - sigla correspondente a variável

\subsection{INSTRUMENTO DE COLETA}

Como instrumento de coleta foi utilizado um questionário, com fulcro no modelo ASH proposto por Quijano e Navarro (1999), e constituído por três módulos. O primeiro contemplou questões referentes aos aspectos sociodemográficos dos servidores técnico-administrativos. $\mathrm{Na}$ segunda seção, os sujeitos da pesquisa apontaram o seu grau de satisfação em relação a 18 atributos. No terceiro módulo, foram instados a mensurar a sua percepção em relação ao nível de importância que a empresa confere a 39 atributos da dimensão cultura organizacional. As respostas, nos dois últimos módulos, foram capturadas por meio de uma escala Likert de 5 pontos, variando de 1 (muito insatisfeito/não é importante) a 5 (muito satisfeito/é muito importante).

\subsection{TRATAMENTO DOS DADOS}

Uma análise fatorial foi desenvolvida, empregando-se o método de extração dos componentes principais e a rotação Varimax, pois consoante Hair et al. (2005), esta técnica estatística é capaz de definir uma estrutura subjacente em um grande número de variáveis, delimitando um conjunto de dimensões latentes comuns, chamadas fatores. Justifica-se o uso em razão da necessidade de identificar as dimensões da satisfação profissional e os estilos de cultura organizacional.

Por fim, o coeficiente de correlação Pearson foi usado para avaliar a magnitude da correlação entre as dimensões da satisfação profissional e os fatores correspondentes aos estilos de cultura organizacional. O tratamento estatístico foi desenvolvido utilizando-se o Statistical Package for the Social Science - SPSS, versão 17.0. A Figura 5 ilustra as técnicas estatísticas usadas na pesquisa, com quais variáveis foram aplicadas e o propósito de sua utilização.

\begin{tabular}{|l|l|c|}
\hline \multicolumn{1}{|c|}{ PROPÓsITOS } & VARIÁVEIS & \multicolumn{1}{|c|}{$\begin{array}{c}\text { FERRAMENTAS } \\
\text { ESTATístICAS }\end{array}$} \\
\hline Identificar as dimensões da satisfação profissional. & De S01 a S18 & Análise Fatorial \\
\hline $\begin{array}{l}\text { Identificar os fatores correspondentes aos estilos da } \\
\text { cultura organizacional. }\end{array}$ & De Q1 a Q39 & Análise Fatorial \\
\hline $\begin{array}{l}\text { Analisar as relações entre as dimensões da satisfação } \\
\text { profissional e dos estilos de cultura organizacional. }\end{array}$ & $\begin{array}{l}\text { De S01 a S18 } \\
\text { De Q1 a Q39 }\end{array}$ & Correlação de Pearson \\
\hline
\end{tabular}

Figura 5 - Técnicas estatísticas utilizadas

Fonte: Elaborado pelos Autores. 


\section{APRESENTAÇÃO E DISCUSSÃO DOS RESULTADOS}

Os resultados aqui apresentados refletem a apuração de 307 respostas válidas. Para alcançar os objetivos propostos, inicialmente, foi utilizada a análise fatorial para identificar as dimensões da satisfação profissional e dos estilos de cultura organizacional. Por fim, foi desenvolvido o teste de correlação de Pearson para verificar o alinhamento (grau de compatibilidade) entre as dimensões da satisfação e da cultura organizacional.

\subsection{IDENTIFICAÇÃO DAS DIMENSÕES DA SATISFAÇÃO PROFISSIONAL}

A análise fatorial aplicada à escala composta pelas 18 variáveis referentes à satisfação profissional, consoante Tabela 2, apresentou valores nos testes $\operatorname{KMO}(0,848)$ e de esfericidade de Bartlett (2585,312 significativo ao nível de 0,000) que reforçam a adequação da amostra à técnica. Quanto às comunalidades de cada uma das variáveis, os índices oscilaram entre 0,518 e 0,913, mostrando que as mesmas explicam adequadamente o fator. Estes escores ratificam os padrões sugeridos por Hair et al. (2005).

Tabela 2 Testes KMO e Bartlett

\begin{tabular}{c|cc}
\hline Medida de adequação de Kaiser-Meyer-Olkin (KMO) & \multicolumn{2}{|c}{0,848} \\
\hline & Aprox. Qui Quadrado & 2585,312 \\
Teste de esfericidade de Bartlett & Graus de liberdade (gl) & 153 \\
& Significância & 0,000 \\
\hline
\end{tabular}

Fonte: Dados da Pesquisa (2012).

As variáveis foram agrupadas em quatro fatores que explicam $62,43 \%$ da variação dos dados originais (Tabela 3). O primeiro fator (F1), nomeado reconhecimento e relacionamento, contemplou sete variáveis, sendo três delas atreladas à relação com os companheiros (S1, S2 e S3), e as outras quatro concernentes à relação com os superiores (S4 e S5) e ao reconhecimento pelo trabalho bem feito (S6 e S7). Percebe-se também como sendo aquele de maior poder de explicação $(36,83 \%)$.

Cinco itens foram carregados no fator 2 (F2), sendo quatro relativos à retribuição (S11 e S12) e à segurança e estabilidade no emprego (S13 e S14), e apenas um referente a benefícios sociais (S17). Este arranjo de variáveis reforça a tendência dos servidores sentirem-se seguros em relação aos seus empregos, provavelmente, por se tratar de uma autarquia federal, onde a estabilidade laboral e a garantia da sua retribuição econômica (salários ou benefícios sociais) são evidentes. Por esse motivo, denominou este fator de segurança e retribuição.

Já o terceiro fator (F3), intitulado autorrealização, foi integrado por quatro variáveis. Dentre eles, a maior parcela era inerentes a autorrealização (S8, S9 e S10), justificando a sua nomenclatura. Por fim, a última dimensão (F4) foi composta apenas por elementos do ambiente físico do trabalho (S15 e S16), daí ter sido chamado de condições físicas. Nota-se ainda que este fator apresentou as cargas fatoriais mais elevadas $(0,882$ e 0,880$)$, demonstrando uma maior correlação de cada variável com o fator (PASQUALI, 1997). Há uma tendência dos servidores sentirem-se mais satisfeitos em relação às condições físicas oferecidas para o exercício das suas 
atividades. Este fenômeno também foi verificado no estudo de Barboza (2008), mesmo este sendo realizado em outro momento da instituição.

Tabela 3 Dimensões extraídas da escala satisfação profissional

\begin{tabular}{|c|c|c|c|c|c|}
\hline Sigla & Variáveis que constituem a escala Satisfação & $\mathbf{F 1}$ & $\mathbf{F 2}$ & F3 & F4 \\
\hline S5 & O respeito que lhe oferecem seus superiores & 0,786 & & & \\
\hline S7 & Os elogios que recebe, quando obtém bons resultados. & 0,780 & & & \\
\hline S1 & O respeito que lhe ofer. os companheiros com os quais trabalha & 0,775 & & & \\
\hline S4 & O apoio que recebe por parte de seus superiores imediatos & 0,743 & & & \\
\hline S2 & As relações que mantém com seus companheiros de trabalho & 0,738 & & & \\
\hline S3 & A possibilidade de fazer amigos no trabalho & 0,689 & & & \\
\hline S6 & O reconhec. que recebe, por parte de seus sup., quando merece & 0,565 & & & \\
\hline S13 & A segurança no emprego, frente ao futuro & & 0,779 & & \\
\hline S14 & O nível de segurança e estabilidade que lhe oferece a empresa & & 0,757 & & \\
\hline S12 & A forma como lhe pagam & & 0,693 & & \\
\hline S11 & O salário que recebe & & 0,593 & & \\
\hline S17 & Os benefícios sociais que a empresa lhe oferece & & 0,357 & & \\
\hline S10 & A adequação de seu trab. à sua form.o de interesses prof. & & & 0,775 & \\
\hline S9 & Nível de conhec. e hab. que pode pôr em jogo no p. de trabalho & & & 0,748 & \\
\hline S8 & A possibilidade de aprender e desenvolver-se profissionalmente & & & 0,717 & \\
\hline S18 & Considerando os aspec. ant., com este emprego você está... & & & 0,444 & \\
\hline S15 & As condições físicas no seu ambiente de trabalho & & & & 0,882 \\
\hline S16 & O ambiente físico de seu posto de trabalho & & & & 0,880 \\
\hline & Percentual da variação explicada (\%) & 36,830 & 9,410 & 8,810 & 7,380 \\
\hline & Percentual acumulado da variação explicada (\%) & 36,830 & 46,240 & 55,050 & 62,430 \\
\hline & Alfa de Cronbach de cada fator & 0,888 & 0,690 & 0,764 & 0,922 \\
\hline & Alfa de Cronbach (escala com 18 variáveis) & \multicolumn{4}{|c|}{0,887} \\
\hline
\end{tabular}

Fonte: Dados da Pesquisa (2012). Legenda: F1- Reconhecimento e relacionamento; F2 - Segurança e retribuição; F3 - Autorrealização; e F4 - Condições físicas.

A fidedignidade dos resultados também foi avaliada a partir dos valores de Alfa de Cronbach encontrados para cada fator, os quais oscilaram de 0,690 a 0,922. Os índices apontam que os 
fatores (dimensões) extraídos mostraram-se confiáveis, conforme preceitua Hair et al. (2005). Em relação à escala satisfação profissional (com 18 itens), o referido indicador apresentou, de forma similar, valor expressivo, atingindo a grandeza de 0,887 . Isto indica a alta confiabilidade do construto, corroborando os achados de Quijano e Navarro (1999).

\subsection{IDENTIFICAÇÃO DAS DIMENSÕES DA CULTURA ORGANIZACIONAL}

Numa primeira aplicação da análise fatorial, observou-se que as variáveis Q9, Q25 e Q30 apresentaram comunalidades inferiores a 0,50. Logo, foram excluídas da análise, consoante pregoa Hair et al. (2005). Uma replicação da técnica permitiu que as variáveis remanescentes fossem agrupadas em seis fatores, que juntos explicam $59,71 \%$ da variância dos dados. Os valores dos testes KMO $(0,898)$ e de esfericidade de Bartlett $(4671,786$ significativo ao nível de 0,000) indicam que o tamanho amostral está adequado à referida ferramenta estatística (Tabela 4). No que tange às comunalidades de cada uma das variáveis, os escores variaram entre 0,506 e 0,777. Isto mostra que elas explicam adequadamente cada fator, segundo os parâmetros sugeridos por Hair et al. (2005).

Tabela 4 Testes KMO e Bartlett

\begin{tabular}{c|cc}
\hline Medida de adequação de Kaiser-Meyer-Olkin (KMO) & \multicolumn{2}{|c}{0,898} \\
& Aprox. Qui Quadrado & 4671,786 \\
Teste de esfericidade de Bartlett & Graus de liberdade (gl) & 630 \\
& Significância & 0,000 \\
\hline
\end{tabular}

Fonte: Dados da Pesquisa (2012).

A Tabela 5 ilustra as dimensões da cultura organizacional. O primeiro fator (F1) foi constituído por 19 itens. Observa-se que, dentre eles, doze contemplam um dos estilos de cultura proposto por Quijano e Navarro (1999), denominado Realização de Pessoas. Nomenclatura, esta, também dada ao primeiro fator da cultura organizacional. O estilo realização de pessoas corresponde a culturas em que se enaltecem as relações interpessoais positivas, a satisfação do coletivo, o estímulo ao crescimento pessoal e ao alcance de metas ambiciosas, porém realistas. No âmbito do IFRN, esta dimensão possui maior ênfase nas relações interpessoais positivas, esperando-se que os seus membros tenham uma atitude amistosa, cooperativa e sensível à satisfação do seu grupo de trabalho (afiliação). Vale enfatizar que esta dimensão revelou um poder de explicação expressivo $(25,079 \%)$ e um elevado Alfa de Cronbach $(0,930)$, denotando a sua confiabilidade e relevância para o estudo.

O segundo fator (F2) encontrado na análise foi formado por seis indicadores (Q34, Q24, Q32, Q6, Q37 e Q19) os quais, em sua totalidade, abrangeu o estilo de cultura ConvencionalDependente. Para Quijano e Navarro (1999), há possibilidade deste estilo está presente nas organizações em que se espera que: os indivíduos relacionem-se com os demais, segundo o modelo habitual de proceder, adaptando-se à ordem estabelecida (convencional-social); as pessoas acatam as ordens, praticamente, sem questioná-las (dependente); ou os membros sentem que devam "estar de acordo com", "obter a aprovação de" e ser querido pelos demais (aprovação). Analisando os elementos intrínsecos a esta dimensão, o IFRN caracterizou-se, 
predominantemente, como uma organização dependente, apesar de também apresentar ínfimos traços de convencionalismo social e aprovação.

A terceira dimensão (F3) contemplou seis variáveis, das quais, cinco, abrangeram o estilo de cultura Poder-Evitação (Q8, Q14, Q38, Q21 e Q12), e por esse motivo, assim foi denominada. As organizações sob esse estilo de cultura mantêm um sistema negativo de recompensas que induz as pessoas a transferirem responsabilidades aos demais e a evitarem a possibilidade de serem culpadas por um erro, desestimulando atitudes de correr riscos e de assumirem novas responsabilidades (evitação). Por outro lado, estimula seus membros a permanecerem atentos aos erros dos demais, opondo-se indiretamente às coisas (oposição), ou, no caso predominante do IFRN, as pessoas são incitadas a obterem fontes de poder, movendo-se politicamente para ganhar influência, sendo, ao mesmo tempo, adaptáveis às demandas dos superiores - poder (QUIJANO; NAVARRO, 1999).

O quarto fator (F4) foi composto por três variáveis (Q23, Q10 e Q11), sendo que dois, dentre eles, remetem ao estilo convencional-tarefa. Por essa razão, foi assim intitulado. Quijano e Navarro (1999) mencionam que tal estilo é marcado por organizações nas quais se espera que se sigam as regras e procedimentos tal como se tem feito sempre. Há uma aversão a implementação de mudanças. Mostra-se uma característica da cultura organizacional do IFRN, realizar tarefas em consonância com os procedimentos habituais, rotineiros; a ponto dos funcionários evitarem opiniões que conflitem as regras e métodos de praxe.

Tabela 5 Dimensões extraídas da escala cultura organizacional

\begin{tabular}{|c|c|c|c|c|c|c|c|}
\hline Sigla & Variáveis que compõem a escala Cultura & $\mathbf{F 1}$ & $\mathbf{F 2}$ & F3 & $\mathbf{F 4}$ & F5 & F6 \\
\hline Q29 & Tenham fácil contato com todos & 0,811 & & & & & \\
\hline Q20 & Pensem em termos de satisfação do grupo & 0,793 & & & & & \\
\hline Q26 & Divulguem suas ideias & 0,774 & & & & & \\
\hline Q16 & Sejam conscientes da realidade da empresa & 0,763 & & & & & \\
\hline Q17 & Sejam "vencedores" & 0,757 & & & & & \\
\hline Q2 & Aj. aos demais a crescer e desenvolverem-se & 0,731 & & & & & \\
\hline Q18 & Prevejam e planejem & 0,724 & & & & & \\
\hline Q33 & Sejam abertos e calorosos & 0,717 & & & & & \\
\hline Q13 & Gostem de seu trabalho & 0,696 & & & & & \\
\hline Q15 & Dediquem tempo aos outros & 0,689 & & & & & \\
\hline Q7 & Tratem os demais de modo agrad. e amistoso & 0,663 & & & & & \\
\hline Q5 & Assumam tarefas que envolvam desafios & 0,638 & & & & & \\
\hline Q39 & Pensem de maneira original e independente & 0,638 & & & & & \\
\hline Q28 & Ajudem os demais a pensar por si mesmos & 0,627 & & & & & \\
\hline Q31 & Assumam riscos altos, embora alcançáveis & 0,602 & & & & & \\
\hline Q35 & Atuem buscando a perfeição & 0,598 & & & & & \\
\hline Q22 & Sejam precisos, mesmo quando não necessário & 0,495 & & & & & \\
\hline Q27 & Questionem hab. decisões tomadas por outros & 0,484 & & & & & \\
\hline Q3 & Se comportem como "bons moços" & 0,424 & & & & & \\
\hline Q34 & Preservem a autoridade sem questioná-la & & 0,764 & & & & \\
\hline Q24 & Aceitem a ordem estabelecida & & 0,715 & & & & \\
\hline Q32 & Obedeçam às ordens de boa vontade & & 0,711 & & & & \\
\hline Q6 & Aceitem os objetivos sem questioná-los & & 0,637 & & & & \\
\hline Q37 & Se adaptem ao "modelo esperado" & & 0,623 & & & & \\
\hline Q19 & Cumpram as ordens, mesmo que sejam equiv.. & & 0,620 & & & & \\
\hline Q8 & Se preocupem em obter poder & & & 0,722 & & & \\
\hline
\end{tabular}




\begin{tabular}{|c|c|c|c|c|c|c|c|}
\hline Q14 & Se oponham indiretamente às coisas & & & 0,717 & & & \\
\hline Q38 & Não gostem de dec. que saem de baixo $p /$ cima & & & 0,639 & & & \\
\hline Q4 & Tendam a competir mais que a colaborar & & & 0,569 & & & \\
\hline Q21 & Se movam "polit." p/ ganhar infl. entre func. & & & 0,561 & & & \\
\hline Q12 & Esperem que os demais ajam primeiro & & & 0,471 & & & \\
\hline Q23 & Não mudem o caminho habitual de proceder & & & & 0,709 & & \\
\hline Q10 & Façam as coisas como sempre fizeram & & & & 0,635 & & \\
\hline Q11 & Adaptem suas opiniões para evitar conflitos & & & & 0,548 & & \\
\hline Q1 & Estejam atentos aos erros dos demais & & & & & 0,836 & \\
\hline Q36 & Se restrinjam às reg. e proced. estabelecidos & & & & & & 0,624 \\
\hline & Percentual da variação explicada (\%) & 25,079 & 17,967 & 6,608 & 3,702 & 3,346 & 3,008 \\
\hline \multicolumn{2}{|r|}{ Percentual acumulado da variação explicada (\%) } & 25,079 & 43,046 & 49,654 & 53,356 & 56,702 & 59,710 \\
\hline \multicolumn{2}{|r|}{ Alfa de Cronbach de cada fator } & 0,930 & 0,831 & 0,768 & 0,698 & 1,00 & 1,00 \\
\hline
\end{tabular}

Fonte: Dados da Pesquisa (2012). Legenda: F1- Realização de Pessoas; F2 - Convencional-Dependente; F3 - PoderEvitação; F4 - Convencional-tarefa; F5 - Oposicionismo; e F6 - Normativo.

Por findar, os demais fatores (F5 e F6) foram constituídos por uma única variável, cada um deles. A quinta dimensão centra-se na subcategoria oposição, componente do estilo poderevitação presente nos estudos de Quijano e Navarro (1999). Esse indicador transmite a ideia de que os membros do IFRN são estimulados a estarem atentos aos erros dos demais, opondo-se indiretamente às coisas. Representa, por conseguinte, uma atitude de "não fazer", de contestar quando se faz ou quando se pretende fazer, existindo, de forma implícita, um desejo de poder. Pelo sentimento retratado neste fator, o mesmo denomina-se oposicionismo. A sexta dimensão contemplou o item Q36, o qual está associado à subcategoria convencional-tarefa. Esta subcategoria refere-se a organizações em que os servidores se limitam a utilizar, apenas, das regras e procedimentos arraigados. Nota-se no IFRN uma tendência em seguir estritamente as normas estabelecidas, implícita ou explicitamente, em virtude dos traços burocráticos impostos pela legislação que disciplina o serviço público brasileiro. Destarte, a execução de tarefas, segundo o procedimento e o costume habitual, é uma característica da cultura organizacional da instituição analisada, e por esse motivo tal fator recebeu a nomenclatura de normativo.

A confiabilidade dos resultados também foi analisada a partir dos valores de Alfa de Cronbach encontrados para cada fator, os quais variaram de 0,698 a 1,00. Os índices confirmam que as dimensões extraídas são confiáveis, consoante apontamentos de Hair et al. (2005). Em relação à escala cultura organizacional (com 39 itens), o Alfa também atingiu um valor expressivo, na ordem de 0,890 . Isto demonstra a alta robustez do construto, corroborando os achados de Quijano e Navarro (1999), Silva (2004) e Barboza (2008).

\subsection{ANÁLISE DAS RELAÇÕES ENTRE AS DIMENSÕES DA SATISFAÇÃO PROFISSIONAL E DA CULTURA ORGANIZACIONAL}

O teste de correlação de Pearson, ao nível de 5 \% de significância, foi empregado com vistas a avaliar a compatibilidade entre as dimensões da satisfação profissional e da cultura organizacional. $\mathrm{O}$ teste foi realizado, considerando pares de dimensões, sendo uma pertencente ao conjunto dos fatores que integram a satisfação e, outra, ao bojo daquelas que compõem a cultura organizacional. A Tabela 6 apresenta os valores dos coeficientes $\left(R_{P}\right)$, apenas para os casos 
em que se detectou uma relação significativa. Nas demais combinações, as hipóteses foram rejeitadas já que não se identificou associação entre o par de fatores avaliado.

Tabela 6 Correlações significativas entre fatores da satisfação e da cultura

\begin{tabular}{c|c|c|c}
\hline $\begin{array}{c}\text { Correlações (Pares de dimensões) } \\
\text { Reconhecimento e Relacionamento x } \\
\text { Estilo Realização de Pessoas }\end{array}$ & Coeficiente de Correlação de Pearson & p-valor & Magnitude \\
\hline $\begin{array}{c}\text { Reconhecimento e relacionamento x } \\
\text { Estilo Poder-Evitação }\end{array}$ & 0,233 & 0.000 & Fraca \\
\hline $\begin{array}{c}\text { Segurança e Retribuição x } \\
\text { Estilo Realização de Pessoas }\end{array}$ & $-0,259$ & 0.000 & Fraca \\
\hline $\begin{array}{c}\text { Autorrealização x } \\
\text { Estilo Realização de Pessoas }\end{array}$ & 0,170 & 0.009 & Fraca \\
\hline $\begin{array}{c}\text { Autorrealização } x \\
\text { Estilo Oposicionismo }\end{array}$ & 0,407 & 0.000 & Moderada \\
\hline $\begin{array}{c}\text { Condições Físicas } x \\
\text { Estilo Convencional-Dependente }\end{array}$ & 0,139 & 0.031 & Fraca \\
\hline
\end{tabular}

Fonte: Dados da Pesquisa (2012). Nota. ${ }^{1}$ Correlação é significante ao nível de 0.05; e ${ }^{2}$ As demais combinações de fatores não apresentaram associação significativa.

Para a interpretação da magnitude das correlações, os Pesquisadores adotaram a seguinte classificação: $R_{P} \leq|0,4|$ (correlação fraca), $|0,4|<R_{P}<|0,7|$ (correlação moderada) e $R_{P} \geq$ $|0,7|$ (correlação forte).

Consta-se que a maior correlação encontrada $(0,407)$, de magnitude moderada, ocorreu entre as dimensões autorrealização (satisfação) e o estilo realização de pessoas (cultura). Ademais, duas outras dimensões da satisfação também estão correlacionadas positivamente com o estilo da cultura denominado realização de pessoas, porém com magnitude fraca. São elas: relacionamento e reconhecimento $(0,233)$; e segurança e retribuição $(0,170)$. Desta forma, apenas a dimensão condições físicas não apresentou relação com o estilo de cultura "realização de pessoas". Isto se justifica pelo fato de, nesse tipo de cultura, os elementos físicos serem irrelevantes, em detrimento as relações interpessoais positivas.

Concernentes aos estilos intitulados convencional-tarefa e normativo, estes, não apresentaram correlação com nenhum dos fatores da satisfação profissional. Em contrapartida, o estilo poder-evitação foi o único que apresentou correlação negativa $(-0,259)$, de magnitude fraca, com a dimensão reconhecimento e relacionamento (satisfação).

Houve ainda uma correlação positiva fraca entre as dimensões condições físicas (satisfação) e convencional-dependente (cultura), na ordem 0,146. Demonstra, assim, que em virtude de as condições físicas de trabalho oferecidas pelo IFRN serem muito satisfatórias, existe uma propensão de que seus servidores técnico-administrativos aceitem, obedeçam e cumpram as ordens de boa vontade, sem questioná-las.

Por último, e com menor grau de correlação positiva fraca $(0,139)$, encontrou-se associação entre a dimensão autorrealização (satisfação) e o estilo de cultura "oposicionismo". 
Nota-se que as quatro dimensões da satisfação estão relacionadas com pelo menos algum estilo de cultura. Assim, é possível afirmar que há relação, mesmo que de magnitude fraca ou moderada, entre a cultura organizacional e a satisfação profissional dos técnicos-administrativos do IFRN, ratificando os apontamentos de Santos e Sustelo (2009). Vale salientar ainda que os baixos valores dos coeficientes de Pearson, constatados na pesquisa, estão em consonância com os achados de Barboza (2008).

\section{CONSIDERAÇÕES FINAIS}

Este artigo propôs uma investigação acerca da relação entre os construtos satisfação e cultura organizacional. Para isto, foi eleito como público-alvo da pesquisa os servidores técnicoadministrativos do Instituto Federal de Educação, Ciência e Tecnologia do Rio Grande do Norte (IFRN), e apreendeu-se, por meio de questionários, as suas percepções sobre um conjunto de variáveis que traduziam as dimensões sobreditas e, ao mesmo tempo, compunham o modelo ASH de Quijano e Navarro (1999).

Dentre os achados, houve a identificação de quatro dimensões da satisfação profissional (reconhecimento e relacionamento; segurança e retribuição; autorrealização; e condições físicas), assim como seis fatores que retratam os estilos de cultura (realização de pessoas; convencionaldependente; poder-evitação; convencional-tarefa; oposicionismo; e normativo). Percebe-se, portanto, uma conformidade entre as dimensões encontradas neste estudo e aquelas presentes nos escritos de Quijano e Navarro (1999) e Barboza (2008).

Ademais, os resultados do teste de Pearson apontaram, mesmo que de magnitude fraca ou moderada, a existência de correlação significativa entre cultura organizacional e a satisfação. Constatou-se que as quatro dimensões da satisfação estão relacionadas com pelo menos algum estilo de cultura - exceto convencional-tarefa e normativo, os quais esta associação não foi evidenciada. A maior correlação encontrada foi entre a dimensão autorrealização (satisfação) e o estilo de cultura intitulado realização de pessoas. A única correlação negativa foi presenciada entre os fatores poder-evitação e, reconhecimento e relacionamento. Os resultados desta pesquisa dão suporte às decisões dos gestores de aperfeiçoamento das estratégias de recursos humanos.

As conclusões deste estudo limitam-se a organização estudada. Sugere-se aos pesquisadores replicá-lo em outros contextos, como, por exemplo, ao envolver instituições privadas; ao estendê-la ao corpo docente da instituição estudada e ao buscar um maior número de organizações participantes, com intuito de investigar a robustez dos achados. Alvitra-se também investigar o sentido da relação entre satisfação e cultura organizacional, com intuito de conhecer qual, dentre elas, exerce a função de antecedente, e aquela que é a consequente.

\section{REFERÊNCIAS}

1. AKTOUF, O. O simbolismo e a cultura de empresa: dos abusos conceituais às lições empíricas. In: CHANLAT, J. F. (Org.). O indivíduo nas organizações: dimensões esquecidas. São Paulo: Atlas, 1994. v. 2, p. 39-79.

2. BARBOZA, J. R. A satisfação profissional e a cultura organizacional: uma análise a partir do modelo de ASH no CEFET. Dissertação de Mestrado em Administração - Universidade Federal do Rio Grande do Norte. Natal, 2008. 
3. BERTERO, C. O. Cultura organizacional e instrumentalização do poder. In: FLEURY, M. T. L.; FISCHER, R. M. Cultura e poder nas organizações. 2. ed. São Paulo: Atlas, 1996.

4. BOURANTAS, D.; ANAGNOSTELIS, J.; MANTES, Y; KAFALAS, A. G. Culture Gap in Greek Management. Organization Studies, 11(2), 261-283, 1990.

5. CAMERON, K. S.; QUINN, R. E. Diagnosing and changing organizational culture. AddisonWesley, 1999.

6. DORNELAS, F. C.; BAPTISTA, J. G. P.; MALACARNE, R.; FREITAS, T. V. Cultura e Poder: um estudo das percepções de atores de diferentes unidades de um mesmo Grupo Industrial. In: ENCONTRO DA ANPAD, 33., 2009, São Paulo. Anais...São Paulo: ANPAD, 2009. p. 2.

7. GIBSON, J. L. et al. Organizações: comportamento, estrutura e processo. São Paulo: McGrawHill, 2006.

8. GIL, A .C. Como elaborar projetos de pesquisa. 5 ed. São Paulo: Atlas, 2010.

9. GOFFEE, R.; JONES, G. The character of a corporation: how your company's culture can make or brake your bussiness. Londres: HarperCollins-Hammersmith, 1998.

10. HAIR, J.F.; ANDERSON, R.E.; TATHAM, R.L.; BLACK, W.C. Análise multivariada de dados. 5 ed. Porto Alegre: Bookman, 2005.

11. HERZBERG, F. The motivation to work. 2. ed. New York: John Wiley \& Sons, Inc., 1964.

12. LÉVI-LEBOYER, C. A crise das motivações. São Paulo: Atlas, 1994.

13. MARTINEZ, M. C. As relações entre a satisfação com aspectos psicossociais no trabalho e a saúde do trabalhador. Dissertação de Mestrado - Faculdade de Saúde Publica, Universidade de São Paulo. São Paulo, 2002.

14. MARTINS M. C. F; SANTOS, G. E. Adaptação e validação de construto da Escala de Satisfação no Trabalho. Revista Psico-USF, v. 11, n. 2, p. 195-205, jul./dez. 2006.

15. OlIVEIRA, J. A. ASH. Auditoria de Sistema Humano. Natal: UFRN/PPGA, 2004a. (Mimeo).

16. OLIVEIRA, S. L. Tratado de metodologia científica: projetos de pesquisa, TGI, TCC, monografias, dissertações e teses. São Paulo: Pioneira Thomson Learning, 2002.

17. PASQUALI, L. Psicometria: teoria e aplicações. Brasília: Universidade de Brasília, 1997.

18. QUIJANO, S.; NAVARRO, J. El ASH (Auditoria del Sistema Humano), los modelos de calidad y evaluación organizativa. Revista de Psicologia General y Aplicada, 1999.

19. ROBBINS, S. P. Comportamento organizacional. São Paulo: Prentice Hall, 2005.

20. SANTOS, J. V.; SUSTELO, M. Cultura organizacional e satisfação profissional: estudo desenvolvido num hospital privado. Revista Psico, v. 40, n. 4, pp. 467-472, out./dez. 2009.

21. SCHEIN, E. H. Organizational culture and leadership. San Francisco: Jossey-Bass, 1985.

22. SILVA, W. S. Diagnóstico Da Cultura Organizacional nos estabelecimentos de panificação de Natal/RN: Uma aplicação do Modelo ASH - Auditoria do Sistema Humano. Dissertação de Mestrado em Administração - Universidade Federal do Rio Grande do Norte, Natal, 2004.

23. VROOM, V. H. Work and motivation. New York: John Wiley, 1964.

24. VERGARA, S. C. Projetos e relatórios de pesquisa em administração. São Paulo: Atlas, 2006. 